Annals of Phlebology

Vol. 18, No. 3, December, 2020

Original Article

https://doi.org/10.37923/phle.2020.18.3.45

\title{
Prevalence of Chronic Venous Disease in Healthcare Workers
}

\author{
'Division of Vascular and Endovascular Surgery, Department of Surgery, Pusan National University Yangsan \\ Hospital, Yangsan, ${ }^{2}$ Division of Vascular Surgery, Department of Surgery, Asan Medical Center, University of \\ Ulsan College of Medicine, Seoul, Korea
}

Su-kyung Kwon, M.D. ${ }^{1}$ and Hyangkyoung Kim, M.D., Ph.D. ${ }^{2}$

\begin{abstract}
Background: Prolonged standing or sitting at work is associated with a high prevalence of chronic venous disease (CVD). We aimed to evaluate the prevalence of CVD by assessing its occurrence and symptoms in hospital health care workers. Methods: We retrospectively analyzed healthcare workers from one university hospital who agreed to take a questionnaire on lower extremity symptoms. The VEINES-QOL/Sym questionnaire was used for clinical evaluation.

Results: A total of 1,166 healthcare workers participated in the survey; $247(21.2 \%)$ were male and $919(78.8 \%)$ were female. The mean age of the participants was $36.3 \pm 10.8(19 \sim 67)$ years. Of the total participants, $237(20.3 \%)$ had no disease, $552(47.3 \%)$ had mild disease, and $377(32.3 \%)$ had moderate to severe disease. The mean (SD) VEINES-QOL/Sym score (score) for all participants was 67.29 (12.38). When sorted by sex, the total scores were significantly lower for female participants than for their male counterparts (female, $64.6 \pm 11.5$ vs. male, $77.3 \pm 10.4, p<0.001)$. The total scores of the nurses/nurse assistants of both sexes were $63.1 \pm 11.5$. The active group score was $77.6 \pm 9.8$, the mid-group score was $70.7 \pm 11.3$, and the sedentary group score was $76.3 \pm 9.4(p<$ 0.001). Linear regression analysis revealed that female sex, age, total working years, and work-related mobility were significantly related to the total score (all, $p<.001$ ).

Conclusion: The prevalence of clinical CVD and venous reflux was high among health care workers. Female sex, nursing vocation, and prolonged standing at work were risk factors for CVD. Prevention and proper management of CVD to improve quality of life should be considered, especially in those groups with high risk factors. (Ann Phlebology 2020;18:45-50)
\end{abstract}

Key Words: Varicose veins, Chronic venous disease, Risk factors, Prevalence, Occupations

Corresponding author : Hyangkyoung Kim, 88 Olympic-ro 43-gil, Songpa-gu, Seoul 05505, Korea, Division of Vascular Surgery, Department of Surgery, Asan Medical Center, University of Ulsan College of Medicine

Tel: 02-3010-1587, Fax: 02-474-9027

E-mail: cindycrow7456@gmail.com

\section{INTRODUCTION}

Chronic venous disease (CVD) is an extremely common venous disorder that affects the lower extremities. The manifestations include a wide range of signs and symptoms, from varicose veins to pain, edema, skin changes, and venous ulcerations. Environmental, behavioral, metabolic, and genetic predisposing factors may induce hemodynamic abnormalities. The development of venous hypertension can lead to venous dilatation and endothelial dysfunction (1). Prolonged venous hypertension caused by vein reflux and chronic inflammation play a central role in the skin changes that occur in the late stages of CVD, which often include chronic venous insufficiency. Chronic inflammation increases the permeability of the venous wall and the movement of leukocytes to the extracellular matrix, which results in symptoms of vein disorders such as swelling, pain, and heaviness $(1,2)$.

Obesity, Deep vein thrombosis (DVT), family history, smoking, female sex, pregnancy, and low physical activity at work have been suggested as potential risk factors for CVD $(3,4)$. Prolonged standing or sitting at work is associated with a high prevalence of varicose veins (5-8). Many healthcare workers are exposed to these risk factors. Due to extended standing times and short walking distances, healthcare workers have a high risk of developing CVD. Prolonged standing or sitting with ambulation for a short distance results in venous pooling of the lower extremities and can eventually induce venous hypertension (8). Studies on the prevalence of CVD in healthcare workers, including physicians and nurses working in hospitals, have been reported in other countries $(8-11)$, but few studies have been conducted in Korea. We aimed to evaluate the prevalence of CVD, related symptoms, and quality of life in hospital health care workers in Korea. 


\section{MATERIALS AND METHODS}

We retrospectively analyzed questionnaires on lower extremity symptoms from health care workers from one university hospital. A total of 1,166 participants, including 247 males and 919 females, completed the survey.

Nurses and nurse assistants were included in one group. Other jobs were divided into three groups according to how much they were moving during the working hours. The group that sat almost all day was classified as sedentary, the group that split the day between sitting and standing was classified as mid, and the group that moved almost all day was classified as active. Based on the CEAP classification, participants were identified as having no CVD (CEAP class $\mathrm{C} 0$ ), mild CVD (CEAP classes $\mathrm{C} 1$ and $\mathrm{C} 2$ ), or moderate to severe CVD (CEAP classes C3, C4, C5, and C6). In order to assess the severity of their own conditions, clinical pictures of various stages of CVD were shown to the participants along with the questionnaire, and patients were categorized into the relevant CEAP classifications according to their responses.

We added the VEINES-QOL/Sym questionnaire to the baseline questionnaire battery. The VEINES-QOL/Sym is a disease-specific patient-reported questionnaire used to evaluate symptoms and quality of life which can cover the entire spectrum of leg-related venous diseases. The VEINESQOL/Sym is a validated tool with reliable psychometric properties for patients with venous leg diseases. The VEINES-QOL/Sym consists of 26 items, 10 of which are symptom-related, nine that assess limitations in daily activities, one that assesses which time of day has the greatest symptom intensity, one that assesses changes during the past year, and five that cover the psychological impacts of venous disease. The questionnaire is self- reported and all responses are rated on 2- to 6-point scales of intensity, frequency, and agreement $(12,13)$. The English version was translated into Korean for our study. We evaluated 21 items; we eliminated the five items about the psychological impact of venous disease. Because the lowest score was not assigned to the most severe symptom in all the questions, some of the questions were reversed in order to obtain consistent results.

We used SPSS version 21.0 software (Armonk, NY, USA) for the statistical analysis. Fisher's exact test or linear by linear association were also used to initially analyze univariate associations of the categorical variables with respect to disease severity. Mean scores between the different groups were analyzed using a t-test or ANOVA. Linear and multilinear analyses with stepwise selections were performed to analyze the association of variables with scores. All p-values $<0.05$ were considered significant.

\section{RESULTS}

Baseline demographics are shown in Table 1. The mean age of the participants was $36.3 \pm 10.8(19 \sim 67)$ years. Male participants were significantly older than female participants (40.72 \pm 10.06 years vs. $35.09 \pm 10.71$ years, $p<.001)$. The working period for men was longer than that for women $(12.93 \pm 10.1$ years vs. $7.14 \pm 8.1$ years, $\mathrm{p}<.001)$. Of the total participants, 237 (20.3\%) had no disease, 552 (47.3\%) had mild disease, and 377 (32.3\%) had moderate to severe disease. None of the patients had a venous ulcer. The mean working period was 8.36 (8.86) years. When categorized by the activity level, working period was $5.8 \pm 6.6$ years for nurses and nurse assistants, $17.1 \pm 10.4$ years for the active group, 10.4 \pm 9.7 years for the mid group, and 12.3 \pm 10.2 years for the sedentary group $(\mathrm{p}<.001)$. Post hoc analysis revealed that the working period was significantly shorter for nurses/nurse assistants and longer in the active group.

The mean (SD) VEINES-QOL/Sym score (score) was 67.29 (12.38) for all participants. When categorized by sex, the total score was significantly lower in female participants

Table 1. Baseline demographics

\begin{tabular}{lcccc}
\hline & Male & Female & Total & $\mathrm{p}$ \\
\hline Number & $247(21.2 \%)$ & $919(78.8 \%)$ & 1166 & $<.001$ \\
Age (year) & $40.72 \pm 10.06$ & $35.09 \pm 10.71$ & & $<.001$ \\
Working duration (year) & $12.93 \pm 10.1$ & $7.14 \pm 8.1$ & $<.001$ \\
Job & & & $694(59.5 \%)$ & \\
Nurse/nurse assistant & $38(15.4 \%)$ & $656(71.4 \%)$ & $92(7.9 \%)$ \\
Active & $70(28.3 \%)$ & $22(2.4 \%)$ & $257(22.4 \%)$ \\
Mid & $89(36.0 \%)$ & $168(18.3 \%)$ & $123(10.5 \%)$ \\
Sedentary & $50(20.0 \%)$ & $73(7.9 \%)$ & \\
\hline
\end{tabular}


(female, 64.6 \pm 11.5 vs. male, 77.3 $\pm 10.4, \mathrm{p}<0.001$ ) (Fig. 1). When the age group was divided into 10 year intervals, the total score was $64.60 \pm 11.9$ for patients aged $\leq 30$ years, $64.9 \pm 12.5$ in patients aged $31 \sim 40$ years, $71.4 \pm 11.5$ in patients aged $41 \sim 50$ years, and $74.1 \pm 10.5$ in patients aged $>50$ years $(p<0.001)$. The total score of the nurse/nurse assistant group was $63.1 \pm 11.5$, the active group score was $77.6 \pm 9.8$, the mid group score was $70.7 \pm 11.3$, and the sedentary group score was $76.3 \pm 9.4(\mathrm{p}<0.001)$. Participants with a working period of $>15$ years had the highest scores $(\mathrm{p}<0.001)$; those with a working period of $0 \sim 5$ years had a total score of $66.7 \pm 12.2$, the group with $6 \sim 10$ years had a score of $64.2 \pm 12.2$, the group with $11 \sim 15$ years had a score of $67.2 \pm 13.6$, and the group with a working period of $<15$ years had a score of $72.8 \pm 10.8$.

Female participants experienced significantly more severe CVD symptoms $(p<.001)$. Younger participants, those with a shorter working period, and nurses/nurse assistants were also associated with more severe CVD symptoms (all, $\mathrm{p}<$ 0.001) (Fig. 2).

Linear regression analysis revealed that female sex, age, working period, and work-related mobility were significantly related to the total score (all, $\mathrm{p}<.001$ ). Multilinear regression analysis showed similar results, except for the active group (all, p<.001) (Table 2).

\section{DISCUSSION}

In this study, the prevalence of CVD based on clinical manifestation and symptoms was $79.7 \%$.

The rate of CVD in our study was higher than that reported in the general population $(39.7 \%$ in men and $32.2 \%$ in women) in the Edinburgh Vein Study (4). The rate of CVD observed among hospital workers in our study was also higher than those reported previously. Based on the CEAP classifications, the reported rate of CVD above the


Fig. 1. Total VEINES-QOL/Sym score. The lower score means the worse symptoms and the lower quality of life. 



Fig. 2. The severity of chronic venous disease. Numbers of participants in each group according to sex, age, job and working period.

C1 level is $34 \% \sim 69 \%(8-10)$. A total of $412(35.3 \%)$ participants in our study presented at least one symptom daily and 915 (78.5\%) experienced symptoms at least once a week.

One of the important findings of our study was that there was a higher prevalence of CVD in females $(89.8 \%)$ than in males $(41.7 \%)$. Even the working duration of males was significantly longer than for females $(12.93 \pm 10.1$ years vs. $7.14 \pm 8.1$ years, $p<.001)$, and the total VEINES-QOL/Sym score was significantly worse for females than males $(64.6 \pm 11.5$ vs. $77.3 \pm 10.4, \mathrm{p}<0.001)$. According to a study on the relationship between long periods of standing and symptoms of CVD, the prevalence of varicose veins was $21.8 \%$ in women and $9.5 \%$ in men. The duration of standing at work was longer in males $(>10$ years, males $50.2 \%$ vs. females $20.5 \%$ ). However, the total standing time per day and consecutive standing hours in the female group were significantly longer than in the male group. The authors determined that the higher prevalence of CVD in females could be affected more by occupational characteristics than by sex itself (7). Nurses and nurse assistants made up $71.4 \%$ of females in our study; $91.4 \%$ 
Table 2. Regression analysis of total score

\begin{tabular}{lcccc}
\hline & Unadjusted & $\mathrm{p}$ & Adjusted & $\mathrm{p}$ \\
\hline Female sex & $-12.674(-14.255 \sim 11.093)$ & $<0.001$ & $-8.426(-10.151 \sim 6.701)$ & $<0.001$ \\
Age & $0.329(0.266 \sim 0.392)$ & $<0.001$ & $0.213(0.139 \sim 0.287)$ & $<0.001$ \\
Working period & $0.234(0.155 \sim 0.314)$ & $<0.001$ & $-0.170(-0.257 \sim 0.082)$ & $<0.001$ \\
Nurse* & $-10.448(-11.767 \sim 9.129)$ & $<0.001$ & $-8.892(-10.859 \sim 6.926)$ & $<0.001$ \\
Active* & $11.174(8.615 \sim 13.734)$ & $<0.001$ & & 0.572 \\
Mid* $^{*}$ & $4.374(2.676 \sim 6.072)$ & $<0.001$ & $-4.954(-6.929 \sim 2.978)$ & $<0.001$ \\
\hline
\end{tabular}

*Reference-sedentary workers.

of whom had CVD, and $41.6 \%$ reported that they had level C3 or C4 CEAP classification. Even though we did not compare nursing with other jobs, its occupational characteristics, or the patient's subjective perceptions of the symptoms, may influence their severity or the patient's quality of life.

Limitations of this study included that no physical examinations were conducted and detailed clinical information was not provided. Because the analysis was based on self-reported results, accurate clinical staging may not have been achieved. However, despite these limitations, the current study has several strengths. For example, overall lower extremity symptoms of hospital workers and their effects on quality of life were investigated. Another strength was that this study was conducted with a large sample size. In addition, it was conducted through a validated questionnaire on venous symptoms in a specific occupational group of health care workers. The VEINES-QOL/Sym questionnaire has been verified for its acceptability, reliability, validity, and responsiveness for venous disease of the lower extremities (12). The results of this study show that the VEINES-QOL/Sym is an excellent tool for screening for CVD in healthcare workers. In this study, young age and female sex were high risk factors, so special consideration for prevention and management should be given to this group.

In conclusion, the prevalence of clinical CVD is high among health care workers. Females, nurses/nurse assistants, and those who stood for prolonged periods of time at work were at higher risk of developing CVD. For subjects with these risk factors, proper screening programs and prevention or proper management is warranted to prevent CVD progression and deterioration in quality of life.

\section{REFERENCES}

1) Ligi D, Croce L, Mannello F (2018) Chronic Venous Disorders: The Dangerous, the Good, and the Diverse. Int
J Mol Sci. 19(9). doi:10.3390/ijms19092544.

2) Bergan JJ, Schmid-Schönbein GW, Smith PDC, Nicolaides AN, Boisseau MR, Eklof BJNEJoM (2006) Chronic venous disease. 355(5):488-498.

3) Criqui MH, Denenberg JO, Bergan J, Langer RD, Fronek A (2007) Risk factors for chronic venous disease: the San Diego Population Study. J Vasc Surg. 46(2):331-337. doi:10.1016/j.jvs.2007.03.052.

4) Robertson LA, Evans CJ, Lee AJ, Allan PL, Ruckley CV, Fowkes FG (2014) Incidence and risk factors for venous reflux in the general population: Edinburgh Vein Study. Eur J Vasc Endovasc Surg. 48(2):208-214. doi:10.1016/j.ejvs.2014.05.017.

5) Tuchsen F, Hannerz H, Burr H, Krause N (2005) Prolonged standing at work and hospitalisation due to varicose veins: a 12 year prospective study of the Danish population. Occup Environ Med. 62(12):847-850. doi:10.1136/oem. 2005.020537.

6) Yun MJ, Kim YK, Kang DM, Kim JE, Ha WC, Jung KY, Choi HW (2018) A Study on Prevalence and Risk Factors for Varicose Veins in Nurses at a University Hospital. Saf Health Work. 9(1):79-83. doi:10.1016/j.shaw.2017.08.005.

7) Bahk JW, Kim H, Jung-Choi K, Jung MC, Lee I (2012) Relationship between prolonged standing and symptoms of varicose veins and nocturnal leg cramps among women and men. Ergonomics. 55(2):133-139. doi:10.1080/00140139. 2011.582957.

8) Cires-Drouet RS, Fangyang L, Rosenberger S, Startzel M, Kidwell M, Yokemick J, McDonald T, Carlin M, Sharma J, Sorkin JD, Lal BK (2020) High prevalence of chronic venous disease among health care workers in the United States. J Vasc Surg Venous Lymphat Disord. 8(2):224-230. doi:10.1016/j.jvsv.2019.10.017.

9) Ziegler SJP (2006) Chronic venous disease is highly prevalent in hospital employees. 13(3):150.

10) Diken AI, Yalcinkaya A, Aksoy E, Yilmaz S, Ozsen K, Sarak T, Cagli K (2016) Prevalence, presentation and occupational risk factors of chronic venous disease in nurses. Phlebology. 31(2):111-117. doi:10.1177/0268355514567491.

11) Huang HK, Weng SF, Su SB, Wang JJ, Guo HR, Hsu CC, Huang CC, Lin HJ (2017) Standing Posture at Work Does Not Increase the Risk of Varicose Veins among Health 
Care Providers in Taiwan. Med Princ Pract. 26(3):266-272. doi:10.1159/000466696.

12) Catarinella FS, Nieman FH, Wittens CH (2015) An overview of the most commonly used venous quality of life and clinical outcome measurements. J Vasc Surg Venous Lymphat Disord. 3(3):333-340. doi:10.1016/j.jvsv.2014.09.003.
13) Lamping DL, Schroter S, Kurz X, Kahn SR, Abenhaim L (2003) Evaluation of outcomes in chronic venous disorders of the leg: development of a scientifically rigorous, patientreported measure of symptoms and quality of life. J Vasc Surg. 37(2):410-419. doi:10.1067/mva.2003.152. 